\title{
Tratamento endovascular de estenose de artéria renal em rim transplantado
}

\section{Endovascular treatment of renal artery stenosis in transplanted kidney}

Francisca Jovita de Oliveira Veras Albuquerque ${ }^{1}$. Rafael Augusto Oliveira Pereira ${ }^{2}$. Carolina Teles de Macedo $^{2}$. João Edison de Andrade Filho ${ }^{3}$. Frederico Augusto de Carvalho Linhares Filho ${ }^{4}$. Carmelo Silveira Carneiro Leão Filho5 .

1 Cirurgiã Geral, Hospital Universitário Walter Cantídio (HUWC), Empresa Brasileira de Serviços Hospitalares (EBSERH), Cirurgiã Vascular, HUWC, médica residente em Cirurgia Vascular, área de atuação Doppler vascular, HUWC, Universidade Federal do Ceará (UFC), Fortaleza, Ceará, Brasil. 2 Estudante de medicina, Universidade Federal do Ceará (UFC), Fortaleza, Ceará, Brasil. 3 Cirurgião vascular, especialista em Endovascular e Doppler Vascular, chefe do Serviço de Cirurgia Vascular, Hospital Universitário Walter Cantídio (HUWC), Fortaleza, Ceará, Brasil. 4 Cirurgião vascular, especialista em Endovascular e Doppler vascular, supervisor da residência médica de Cirurgia Vascular, Hospital Universitário Walter Cantídio (HUWC), Fortaleza, Ceará, Brasil. 5 Mestre em Cirurgia pela Universidade Federal do Ceará, cirurgião vascular, preceptor da residência de Cirurgia Vascular, Hospital Universitário Walter Cantídio (HUWC), Universidade Federal do Ceará (UFC), Fortaleza, Ceará, Brasil.

\section{RESUMO}

Introdução: a estenose de artéria renal transplantada (EART) é a complicação vascular mais frequente do transplante renal. O diagnóstico é realizado através da arteriografia e o tratamento consiste na angioplastia transluminal percutânea (ATP). Objetivo: avaliar o sucesso clínico, a patência primária e secundária da ATP, bem como a sobrevida dos pacientes após o tratamento endovascular. Métodos: análise retrospectiva dos pacientes com EART tratados por ATP no período de janeiro de 2008 a dezembro de 2016 no Hospital Universitário Walter Cantídio. Resultados: 28 pacientes foram tratados por EART através do método endovascular. A pressão diastólica reduziu de $91,62 \mathrm{mmHg}$ para $76 \mathrm{mmHg}$, em média, após 6 meses do tratamento $(\mathrm{p}<0,05)$. Observou-se uma redução nos níveis de creatinina após 3, 6 e 24 meses da angioplastia $(\mathrm{p}=0,005)$. As taxas de patência primária em 1, 3, 6, 12 e 24 meses foram de 89,3\% +/- 5,8\%, 85,6\% +/- 6,7\%, 81,8\% +/- 7,4\%, 78,1\% +/-7,9\% e 68,9\% +/- 9,3\%, respectivamente. A taxa de patência secundária foi de $100 \%$. A taxa de sobrevida em 1, 3, 6, 12 e 24 meses foi de $96,4 \%+/-3,5 \%$, $96,4 \%+/-3,5 \%, 92,6 \%+/-5,1 \%, 92,6 \%+/-5,1 \%$ e $83,8 \%+/-7,5 \%$, respectivamente. Conclusão: o tratamento endovascular de EART apresenta boas taxas de patências primária e secundária e é efetivo em restaurar e manter a função renal em dois anos.

Palavras-chave: Transplante de rim. Obstrução da artéria renal. Angioplastia. Sobrevida.

\begin{abstract}
Introduction: Transplant renal artery stenosis (TRAS) is the most frequent vascular complication of renal transplantation. The diagnosis is made through arteriography and the treatment consists of percutaneous transluminal angioplasty (PTA). Objective: to evaluate the clinical success, the primary and secondary patency of PTA, as well as the survival of patients after endovascular treatment. Methods: Retrospective analysis of patients with TRAS treated by PTA from January 2008 to December 2016 at Hospital Universitário Walter Cantídio. Results: 28 patients were treated by TRAS using the endovascular method. Diastolic pressure decreased from $91.62 \mathrm{mmHg}$ to $76 \mathrm{mmHg}$, on average, after 6 months of treatment $(\mathrm{p}<0.05)$. There was a decrease in creatinine levels after 3,6 and 24 months of angioplasty $(p=0.005)$. The primary patency rates at $1,3,6,12$ and 24 months were $89.3 \%+/-5.8 \%, 85.6 \%+/-6.7 \%, 81.8 \%+/-7,4 \%, 78.1 \%+/-7.9 \%$ and $68.9 \%+/-9.3 \%$, respectively. The secondary patency rate was $100 \%$. The survival rate at $1,3,6,12$ and 24 months was $96.4 \%+/-3.5 \%, 96.4 \%+/-3.5 \%, 92.6 \%+/-5,1 \%, 92.6 \%+/-5.1 \%$ and $83.8 \%+/-7.5 \%$, respectively. Conclusion: Endovascular treatment of TRAS presents good rates of primary and secondary patency and is effective in restoring and maintaining renal function in two years.
\end{abstract}

Keywords: Kidney transplantation. Renal artery obstruction. Angioplasty. Survivorship.

Autor correspondente: Francisca Jovita de Oliveira Veras Albuquerque, Rua Nunes Valente, 275, apartamento 1001, Meireles, Fortaleza, Ceará. CEP: 60125-070. Telefone: +55 85 98839-3029. E-mail: jovitaveras@gmail.com

Conflito de interesses: Não há qualquer conflito de interesses por parte de qualquer um dos autores.

Recebido em: 07 Nov 2017; Revisado em: 21 Nov 2017; Aceito em: 21 Nov 2017. 


\section{INTRODUÇÃO}

A estenose de artéria renal transplantada (EART) é a mais frequente complicação vascular do transplante renal, com uma incidência variando de 1 a 23\%, de acordo com a definição e a técnica diagnóstica utilizada. ${ }^{1}$ Pacientes com essa patologia podem apresentar hipertensão refratária e/ou aumento dos níveis de creatinina, geralmente entre 3 meses a 2 anos após o transplante, embora apresentações precoces ou tardias possam ocorrer.

Os principais fatores de risco de EART incluem doença aterosclerótica no rim doador ou nas artérias receptoras, fatores imunológicos, trauma durante a cirurgia e anormalidades anatômicas no enxerto. ${ }^{2}$ Pode ser dividida de acordo com a localização da estenose em pré-anastomótica, anastomótica ou pós-anastomótica. Ocasionalmente, o estreitamento pode acometer simultaneamente vários segmentos ou mesmo todo o vaso. ${ }^{3}$

Os exames de triagem para EART incluem US Doppler e angioressonância, sendo a arteriografia o padrão-ouro para o diagnóstico definitivo. ${ }^{4}$ Angioplastia transluminal percutânea (ATP) é o tratamento inicial de escolha para EART, com ou sem colocação de stent. Estudos indicam que a angioplastia com stent primário pode retardar ou prevenir recorrências de EART, evitando-se, dessa forma, a necessidade de punções arteriais repetidas, a realização de angiografias adicionais e a possibilidade de complicações. ${ }^{5}$

O objetivo desse estudo é analisar a patência primária e secundária da ATP com ou sem colocação de stent para o tratamento da EART no Hospital Universitário Walter Cantídio - Universidade Federal do Ceará.

\section{METODOLOGIA}

Foi realizado um estudo retrospectivo de todos os pacientes com EART tratados entre janeiro de 2008 e dezembro de 2016 no Hospital Universitário Walter Cantídio-UFC. Através de revisão de prontuários foram coletados dados como idade, gênero, características do órgão doador, tipo de anastomose, apresentação clínica, tempo de surgimento dos sintomas após o transplante, tipo de procedimento (angioplastia com ou sem colocação de stent, tipo e número de stents), valores de creatinina, níveis pressóricos e número de drogas anti-hipertensivas antes e após o procedimento.

O diagnóstico de EART foi suspeitado clinicamente através do súbito aparecimento de hipertensão ou a rápida progressão dos níveis pressóricos e/ou aumento dos níveis de creatinina. Os pacientes foram submetidos a US Doppler do enxerto, sendo os seguintes achados considerados indicadores significantes de estenose de artéria renal: pico de velocidade sistólica $($ VPS $)>200 \mathrm{~cm} / \mathrm{s}$, índice de resistência $<0,5$ e gradiente de velocidade $>2: 1$.

A artéria renal transplantada foi implantada na artéria ilíaca comum ou externa através de anastomose término-lateral em todos os pacientes. O procedimento endovascular foi realizado com anestesia local e sedação, com punção retrógrada da artéria femoral ipsilateral ao enxerto, sendo utilizado um introdutor curto 5F. Após passagem do introdutor, os pacientes receberam heparina não fracionada na dose de $80 \mathrm{U} /$ $\mathrm{kg}$. Uma angiografia não seletiva foi realizada inicialmente para confirmação do diagnóstico, sendo tratados os pacientes que apresentaram estenose maior que 70\%. Angioplastia transluminal percutânea com stent primário foi o tratamento inicial de escolha para a maioria dos pacientes.

Seguimento de 1, 3, 6, 12 e 24 meses após o tratamento endovascular incluiu medidas de pressão sanguínea, avaliação do número de drogas anti-hipertensivas, níveis de creatinina e US Doppler. Na suspeita de reestenose da artéria renal foi realizado nova angiografia para confirmação do diagnóstico e tratamento endovascular quando possível.

Foi utilizado a curva de Kaplan-Meier para representar as taxas de patência e sobrevida. Um valor de $\mathrm{p}<0,05$ foi considerado estatisticamente significante. O programa de estatística SPSS versão 22, IBM, USA, foi utilizado para análise dos dados, através dos testes de Friedman e Wilcoxon.

\section{RESULTADOS}

No período de janeiro de 2008 a dezembro de 2016 foram realizados 819 transplantes renais no Hospital Universitário Walter Cantídio, sendo 28 pacientes tratados por estenose de artéria renal no rim transplantado (3,4\%). Dentre os acometidos, 16 eram homens e 12 eram mulheres, com uma média de idade de 46,7 anos (intervalo, 32 a 67 anos). O enxerto renal foi de doadores falecidos em quase todos os pacientes, sendo somente um proveniente de doador vivo.

Em relação à apresentação clínica de EART, hipertensão de início súbito foi encontrada em quatro pacientes, aumento dos níveis de creatinina em três pacientes, função retardada do enxerto em três casos, ambos hipertensão e aumento dos níveis de creatinina em 15 pacientes e anormalidades severas no US Doppler do enxerto, sem manifestações clínicas, em três pacientes. A média de tempo entre o transplante renal e o surgimento de alterações clínicas foi 396, 3 dias (intervalo, 1 a 3358 dias). Um paciente apresentou estenose importante da artéria renal no pós-operatório imediato, recebendo tratamento endovascular uma semana após o transplante renal (Figuras 1A e 1B).

A localização da estenose da artéria renal foi ostial $(n=9)$, justa-ostial $(n=12)$ e no terço médio $(n=7)$. Em três pacientes a estenose foi devido a uma torção da artéria distal à anastomose, sendo prontamente resolvida após a colocação do stent. Três pacientes foram tratados apenas com angioplastia por balão em um primeiro momento, sendo necessário, posteriormente, nova angioplastia com aposição de stent. Os demais realizaram angioplastia com stent primário não revestido, balão-expansível e com sistema de rápida troca. Foram utilizados 36 stents: 11 Tsunami Peripheral (Terumo), 9 Pro-Kinetic Energy (Biotronik), 7 Dynamic Renal 
(Biotronik), 6 Apolo 3 (Cordynamic), 2 Mustang Coronary (MicroPort) e um Herculink Elite (Abbott Vascular). O diâmetro médio do material utilizado foi $4,9 \mathrm{~mm}$ (intervalo, 3 a $7 \mathrm{~mm}$ ) e a média de comprimento foi $18,3 \mathrm{~mm}$ (intervalo, 12 a $26 \mathrm{~mm}$ ). Em dois casos observou-se artéria renal dupla com estenoses justa-ostiais em ambas, sendo tratadas através de angioplastia com stent (Figuras 2A e 2B). Um paciente realizou kissing stent para tratamento de estenose em bifurcação de ramos da artéria renal. Nenhuma complicação intra-operatória foi observada. A eficácia da angioplastia percutânea da EART foi imediatamente verificada através de angiografia de controle. A taxa de sucesso técnico foi de $100 \%$.

Figura 1. (A) Estenose ostial de artéria renal transplantada; (B) Tratamento endovascular de EART bem sucedido.
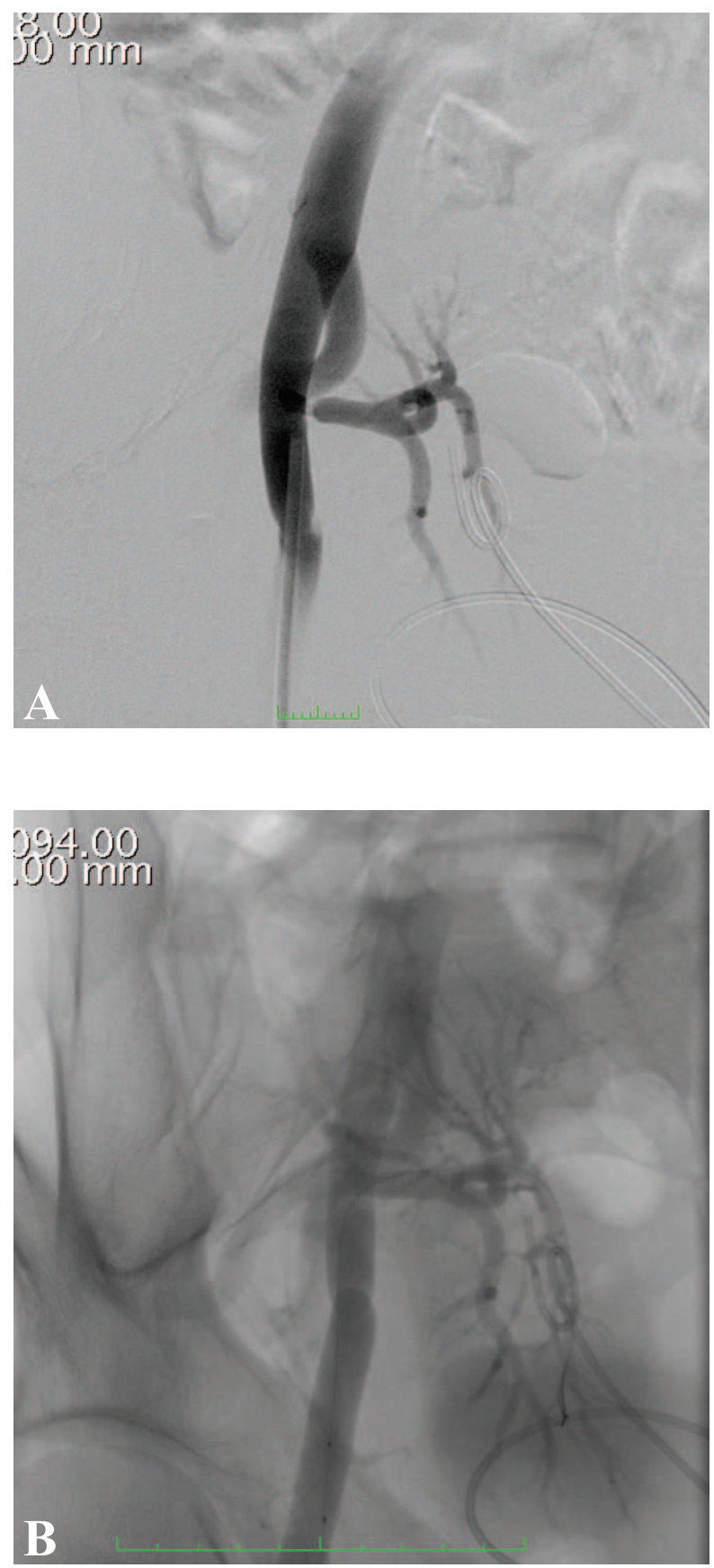

Figura 2. (A) Estenose em artéria renal dupla; (B) Tratamento endovascular com colocação de stent.
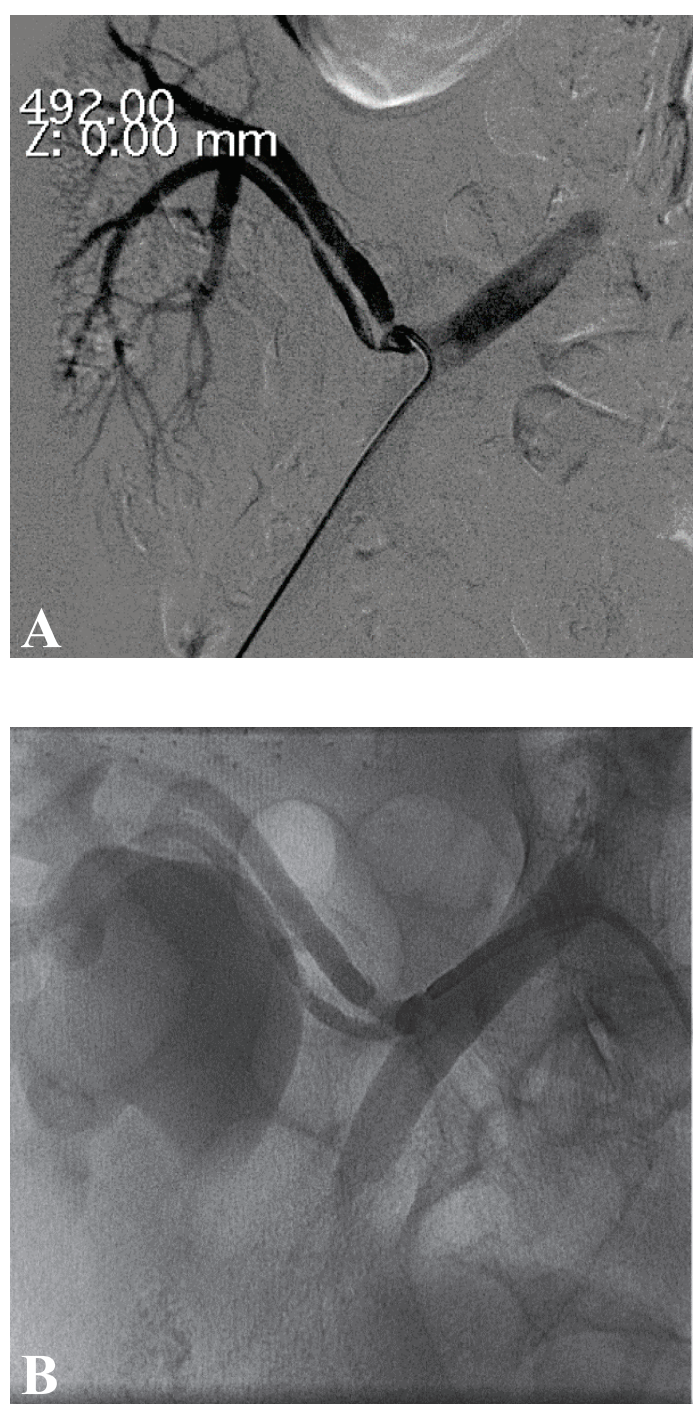

\section{Resultados pós-operatórios imediatos e tardios}

A diminuição dos níveis de pressão sistólica após a terapia endovascular não mostrou resultado estatisticamente significante durante o seguimento $(\mathrm{p}=0,08)$. A pressão diastólica reduziu de $91,6 \mathrm{mmHg}$ (variação, $60-160 \mathrm{mmHg}$ ) para 80,5 $\mathrm{mmHg}$ (variação, $60-100 \mathrm{mmHg}$ ) no pós-operatório imediato; $77,4 \mathrm{mmHg}$ (variação, 60-100mmHg) após 1 mês e $76 \mathrm{mmHg}$ (variação, 60-90mmHg) após 6 meses da angioplastia, com resultado estatisticamente significante $(\mathrm{p}<0,05)$. O número de drogas antihipertensivas durante o acompanhamento não mostrou diferença significativa quando comparada aos valores pré-operatórios ( $\mathrm{p}=0,085)$ (Gráficos 1 e 2).

Em relação aos valores pré-tratamento, observou-se uma redução importante nos níveis de creatinina após 3, 6 e 24 meses da angioplastia ( $\mathrm{p}=0,005)$, passando de $2,4 \mathrm{mg} / \mathrm{dl}$ (variação, 0,5 - 10,1mg/dl) para 1,37 mg/dl (variação, 0,6 $4,0 \mathrm{mg} / \mathrm{dl}$ ) no $3^{\circ}$ mês, $1,35 \mathrm{mg} / \mathrm{dl}$ (variação, $0,6-3,2 \mathrm{mg} / \mathrm{dl}$ ) no $6^{\circ}$ mês e $1,23 \mathrm{mg} / \mathrm{dl}$ (variação, $0,5-2,9 \mathrm{mg} / \mathrm{dl}$ ) após 24 meses do tratamento (Gráfico 3). 
Gráfico 1. Seguimento e comparação das pressões sistólica e diastólica.

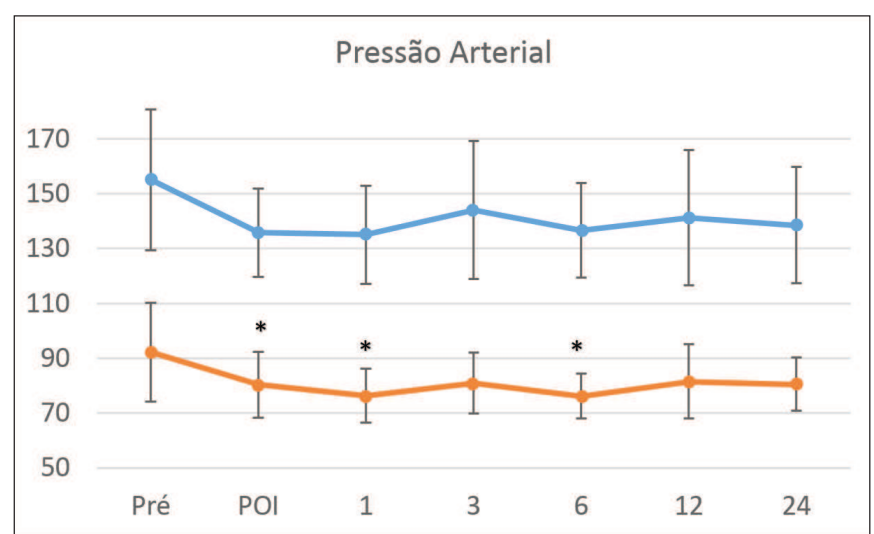

Azul: PA sistólica; $\mathrm{p}=0,08$; Laranja: PA diastólica; * $\mathrm{p}<0,05$ versus pré.

Gráfico 2. Seguimento e comparação do número de drogas antihipertensivas prescritas aos pacientes pré e pós ATP.

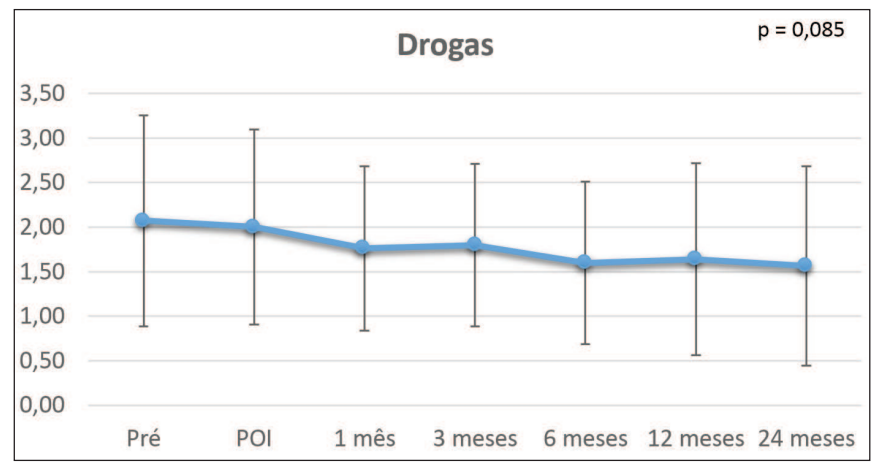

Gráfico 3. Seguimento e comparação do valor de creatinina pré e pós ATP.

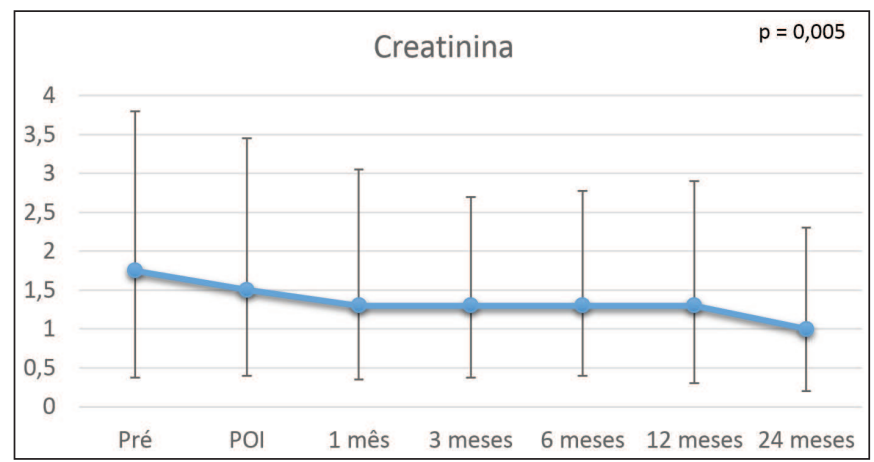

${ }^{*} \mathrm{p}<0,05$ versus valor pré

\section{Patência e Sobrevida}

As taxas de patência primária em 1, 3, 6, 12 e 24 meses (IC $+/-95 \%$ ) foram de $89,3 \%+/-5,8 \%, 85,6 \%+/-6,7 \%, 81,8 \%$ $+/-7,4 \%, 78,1 \%+/-7,9 \%$ e $68,9 \%+/-9,3 \%$ respectivamente (Gráfico 4). A taxa de patência secundária foi de $100 \%$, com ausência de estenose recorrente no período de seguimento.

Houve reestenose em sete pacientes, três dos quais foram tratados inicialmente com angioplastia com balão, como descrito anteriormente. Os outros quatro pacientes haviam sido tratados inicialmente com stent primário, sendo necessário nova intervenção com colocação de stent em três casos.

A taxa de sobrevida foi de $96,4 \%+/-3,5 \%$ no primeiro mês, $96,4 \%+/-3,5 \%$ em 3 meses, $92,6 \%+/-5,1 \%$ em 6 meses, $92,6 \%$ $+/-5,1 \%$ em 1 ano e 83,8\% +/- 7,5\% em 2 anos (Gráfico 5).

Gráfico 4. Curva de Kaplan-Meier para patência primária.

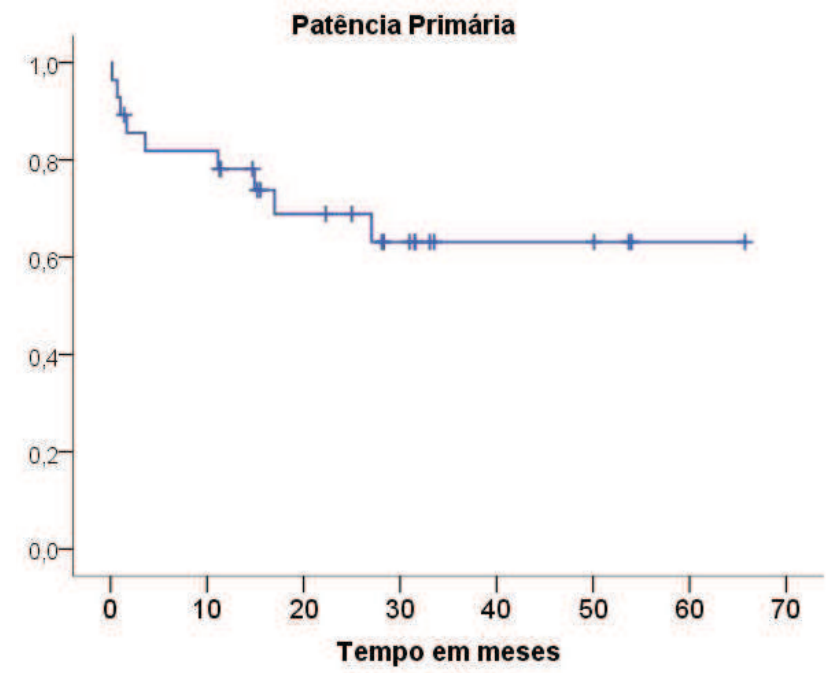

Gráfico 5. Curva de Kaplan-Meier para sobrevida.

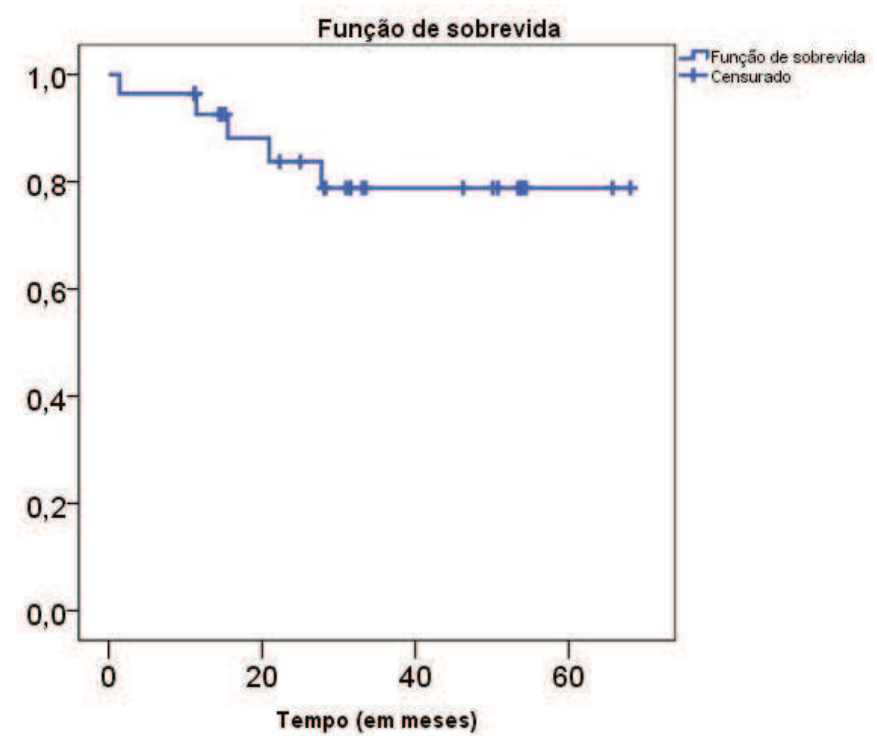

Ocorreram cinco óbitos, sendo três pacientes admitidos na emergência em parada cárdio respiratória sem causa definida, um faleceu por infarto agudo do miocárdio e outro por AVC isquêmico 42 dias após o procedimento endovascular. As mortes aconteceram em média 13,6 meses (variação, 1,4 27,7 meses) após a ATP. Três pacientes evoluíram com perda do enxerto renal, dois por rejeição crônica - 15 e 16 meses após o tratamento, e um por trombose da artéria renal - 13,2 meses após a angioplastia.

O tempo médio de seguimento foi 20,1 meses (variação, 1,4 -24 meses). 


\section{DISCUSSÃO}

A EART é uma séria complicação do transplante renal, cuja incidência tem uma ampla variação, de 1 a $23 \%$, devido às diferentes modalidades de diagnóstico. ${ }^{6} \mathrm{O}$ estudo publicado por Hurst et al. ${ }^{7}$ em 2009, através do registro do Sistema de Dados Renais dos Estados Unidos (USRDS), encontrou uma incidência cumulativa de EART de $2 \%$ em 3 anos e uma taxa de incidência geral de 8,3 casos por 1000 pacientes-ano (IC $95 \% 7,8-8,9)$. A incidência na nossa amostra foi de 3,4\%, taxa semelhante à da literatura.

Geralmente a EART ocorre entre 2 meses a 3 anos após o transplante renal, com uma alta incidência nos primeiros seis meses. ${ }^{7} \mathrm{O}$ surgimento precoce da estenose é principalmente devido à injúria intimal traumática durante a retirada ou manipulação do vaso, acotovelamento da artéria quando esta é mais longa que a veia ou por problemas técnicos com a sutura vascular, enquanto estenoses que ocorrem tardiamente refletem hiperplasia da artéria renal do enxerto ou evolução da aterosclerose da artéria renal e/ou ilíaca. ${ }^{8}$ A média de tempo entre o transplante renal e a apresentação clínica encontrada por nós foi de 396,3 dias, sendo que $67,8 \%$ (19/28) dos pacientes desenvolveram sintomas de EART nos primeiros 6 meses após o transplante.

Fatores de risco relacionados com EART incluem: critérios expandidos de doadores (principalmente a idade), obesidade e doença coronariana. ${ }^{7}$ Estudos relatam taxas de prevalência de EART em transplantes de doadores falecidos de 4,1\%, 4,5\% e $6,5 \%$ comparado com $0,3 \%, 0,8 \%$ e $1,7 \%$ em transplantes de doadores vivos. ${ }^{6}$ Entretanto, outras pesquisas afirmam não haver correlação significativa entre o tipo de doador e a estenose de artéria renal transplantada. ${ }^{9}$

Os indicadores clínicos de EART são hipertensão de início súbito ou refratária e/ou disfunção do enxerto, com aumento dos níveis de creatinina. A presença dessas alterações deve ser a principal indicação de tratamento. US Doppler, isoladamente, evidenciando estenose proximal do enxerto renal nem sempre implica em consequência clínica. Siskind et al. ${ }^{10}$ descreveram alterações severas da VPS $(>400 \mathrm{~cm} / \mathrm{s})$ em aproximadamente $45 \%$ dos pacientes transplantados com evolução clínica normal ou normalização da velocidade de pico sistólica, na maioria dos casos, sem intervenção. Somente aqueles que persistem com valores elevados de VPS são direcionados para realização de arteriografia e tratamento quando necessário.

Existem dois tipos de abordagem terapêutica: tratamento cirúrgico e endovascular. A cirurgia está indicada em casos de falha ou impossibilidade do tratamento endovascular, devido ao alto risco de complicações como perda do enxerto em 15 a $20 \%$ dos casos, trauma ureteral e reoperação. ${ }^{11}$ A grande maioria dos casos de EART são tratados pelo método endovascular, preferencialmente através de angioplastia com colocação de stent primário, uma vez que há diminuição na taxa de reestenose e menores índices de complicações. ${ }^{12}$

A EART geralmente acomete a anastomose cirúrgica, contudo podem ser pré ou pós-anastomóticas. ${ }^{13}$ Apesar da menor efetividade e maior risco da angioplastia transluminal percutânea em lesões na linha de sutura, ${ }^{11}$ muitos autores têm reportado ausência de complicações nesse local, com o procedimento endovascular sendo realizado, pelo menos, 2 meses após o transplante. ${ }^{14}$ Em nossa série, nove lesões estavam localizadas na anastomose e o restante após a linha de sutura (sete lesões justa-ostiais e 12 no terço médio). Não foi observado nenhuma complicação do tratamento endovascular quando realizado em estenoses ostiais, incluindo o procedimento feito após uma semana do transplante renal.

A abordagem endovascular para EART é uma técnica segura, com índices de complicações variando entre $0 \%{ }^{15,16}$ a $4,5 \%{ }^{3}$ e com elevadas taxas de sucesso técnico, frequentemente relatadas entre $88 \%{ }^{3,17}$ e $100 \% .^{15,16}$ Obtivemos taxas de $0 \%$ e $100 \%$ para complicações e sucesso do procedimento, respectivamente.

Reestenose representa uma das principais complicações do método endovascular, particularmente quando realizado somente através de angioplastia com balão, com taxas de reestenose entre $10 \%$ a $56 \% .{ }^{9,18}$ Diversos estudos têm relatado taxas menores, menos de $10 \%$, quando realizado angioplastia com stent primário. ${ }^{19}$ Observou-se reestenose em 100\% dos pacientes tratados somente com angioplastia por balão $(3 / 3)$ e em $16 \%$ dos pacientes que realizaram colocação de stent na primeira abordagem $(4 / 25)$.

Não há consenso em relação à efetividade do tratamento endovascular na melhora da hipertensão e redução de drogas anti-hipertensivas. Algumas séries publicadas relatam diminuição significativa nos valores de pressão arterial, bem como no número de medicações anti-hipertensivas, ${ }^{19,8}$ enquanto outras obtêm resultados sem significância estatística. ${ }^{20} \mathrm{Em}$ nosso estudo a maioria desses resultados não foi estatisticamente significante. O impacto da ATP nos níveis de creatinina pós-operatória parece ser mais consensual, com a maioria dos relatos mostrando uma redução importante após o tratamento, resultado também observado por nós. ${ }^{14,16}$

\section{CONCLUSÃO}

O tratamento endovascular de EART com stent primário mostra-se seguro e com altas taxas de sucesso técnico. Apresenta boas taxas de patências primária e secundária e é efetivo em restaurar e manter a função renal de enxertos renais. Os resultados relacionados com os níveis de pressão arterial e número de drogas anti-hipertensivas necessitam ser melhor avaliados através de estudos prospectivos e randomizados.

\section{REFERÊNCIAS}

1. Fervenza FC, Lafayette RA, Alfrey EJ, Petersen J. Renal artery stenosis in Kidney transplants. Am J Kidney Dis. 1998;31(1):142-8.
2. Roberts JP, Ascher NL, Fryd DS, Hunter DW, Dunn DL, Payne WD, et al. Transplant renal artery stenosis. Transplantation. 1989;48(4):580-3. 
3. Marini M, Fernandez-Rivera C, Cao I, Gulias D, Alonso A, LopezMuniz A, et al. Treatment of transplant renal artery stenosis by percutaneous transluminal angioplasty and/or stenting: study in 63 patients in a single institution. Transplant Proc. 2011;43(6):2205-7.

4. Beecroft JR, Rajan DK, Clark TW, Robinette M, Stavropoulos SW. Transplant renal artery stenosis: outcome after percutaneous intervention. J Vasc Interv Radiol. 2004;15(12):1407-13.

5. Ridgway D, White SA, Nixon M, Carr S, Blanchard K, Nicholson ML. Primary endoluminal stenting of transplant renal artery stenosis from cadaver and non-heart-beating donor kidneys. Clin Transplant. 2006;20:394-400

6. Patel NH, Jindal RM, Wilkin T, Rose S, Johnson MS, Shah H, et al. Renal arterial stenosis in renal allografts: retrospective study of predisposing factors and outcome after percutaneous transluminal angioplasty. Radiology. 2001;219(3):663-7.

7. Hurst FP, Abbott KC, Neff RT, Elster EA, Falta EM, Lentine KL, et al. Incidence, predictors and outcomes of transplant renal artery stenosis after kidney transplantation: analysis of USRDS. Am J Nephrol. 2009;30(5):459-67.

8. Becker BN, Odorico JS, Becker YT, Leverson G, McDermott JC, Grist T, et al. Peripheral vascular disease and renal transplant artery stenosis: a reappraisal of transplant renovascular disease. Clin Transplant. 1999;13(4):349-55.

9. Audard V, Matignon M, Hemery F, Snanoudj R, Desgranges P, Anglade MC, et al. Risk factors and long-term outcome of transplant renal artery stenosis in adult recipients after treatment by percutaneous transluminal angioplasty. Am J Transplant. 2006;6(1):95-9.

10. Siskind E, Lombardi P, Blum M, Tyrell R, Villa M, Kuncewitch $\mathrm{M}$, et al. Significance of elevated transplant renal artery velocities in the postoperative renal transplant patient. Clin Transplant. 2013;27(2):e157-60.

11. Merkus JW, Huysmans FT, Hoitsma AJ, Buskens FG, Skotnicki $\mathrm{SH}$, Koene RA. Renal allograft artery stenosis: results of medical treatment and intervention. A retrospective analysis. Transpl Int. 1993;6(2):111-5.

12. Valpreda S, Messina M, Rabbia C. Stenting of transplant renal artery stenosis: outcome in a single center study. J Cardiovasc Surg (Torino). 2008;49(5):565-70.

13. Lacombe M. Arterial stenosis complicating renal allotransplantation in man. Ann Surg. 1975;181(3):283-8.

14. Sharma S, Potdar A, Kulkarni A. Percutaneous transluminal renal stenting for transplant renal artery stenosis. Catheter Cardiovasc Interv. 2011;77(2):287-93.

15. Su CH, Lian JD, Chang HR, Wu SW, Chen SC, Tsai CF, et al. Long-term outcomes of patients treated with primary stenting for transplant renal artery stenosis: a 10-year case cohort study. World J Surg. 2012;36(1):222-8.

16. Pappas P, Zavos G, Kaza S, Leonardou P, Theodoropoulou E, Bokos J, et al. Angioplasty and stenting of arterial stenosis affecting renal transplant function. Transplant Proc. 2008;40(5):1391-6.

17. Touma J, Costanzo A, Boura B, Alomran F, Combes M. Endovascular management of transplant renal artery stenosis. J Vasc Surg. 2014;59(4):1058-65.

18. Raynaud A, Bedrossian J, Remy P, Brisset JM, Angel CY, Gaux JC, et al. Percutaneous transluminal angioplasty of renal transplant renal artery stenosis. AJR Am J Roentgenol.1986;146(4):853-7.

19. Voiculescu A, Schmitz M, Hollenbeck M, Braasch S, Luther B, Sandmann W, et al. Management of arterial stenosis affecting kidney graft perfusion: a single-centre study in 53 patients. Am J Transplant. 2005;5(7):1731-8.

20. Geddes CC, McManus SK, Koteeswaran S, Baxter GM. Long-term outcome of transplant renal artery stenosis managed conservatively or by radiological intervention. Clin Transplant. 2008;22(5):572-8.

\section{Como citar:}

Albuquerque FJ, Pereira RA, Macedo CT, Andrade JE Filho, Linhares FA Filho, Leão CS Filho. Tratamento endovascular de estenose de artéria renal em rim transplantado. Rev Med UFC. 2017 set-dez;57(3):35-40. 